\title{
Coarsely Calibrated Visual Servoing of a Mobile Robot using a Catadioptric Vision System
}

\author{
Romeo Tatsambon Fomena, Han Ul Yoon, Andrea Cherubini, François Chaumette and Seth Hutchinson
}

\begin{abstract}
A catadioptric vision system combines a camera and a mirror to achieve a wide field of view imaging system. This type of vision system has many potential applications in mobile robotics. This paper is concerned with the design of a robust image-based control scheme using a catadioptric vision system mounted on a mobile robot. We exploit the fact that the decoupling property contributes to the robustness of a control method. More precisely, from the image of a point, we propose a minimal and decoupled set of features measurable on any catadioptric vision system. Using the minimal set, a classical control method is proved to be robust in the presence of point range errors. Finally, experimental results with a coarsely calibrated mobile robot validate the robustness of the new decoupled scheme.
\end{abstract}

\section{INTRODUCTION}

There is currently a growing interest in using omnidirectional vision system for mobile robotics [1]. An important advantage of omnidirectional vision systems over classical perspective cameras is that the former do not suffer from a restricted field of view (FOV). This can facilitate landmark visibility during mobile robot navigation.

Vision-based control of a mobile robot can be applied to pose stabilization, visual homing and path following. These applications can require the robot to localize itself in the environment. Based on the localization approach, several control techniques have been proposed. To keep a robot on a desired or predefined path, a homography between the reference and the current image can be used to define adequate visual features for navigation [2]. It is also possible to exploit the epipolar geometry between the reference and the current image to drive the robot to a desired position [3]. An image memory can be used to guide the robot on a pretaught path [4]. In this last application, Cartesian coordinates of a point centroid have been used as features in the visual servoing scheme.

Other geometric features have been proposed for robot visual navigation. A normalized cross correlation can be used to control the robot heading [5]. The robot can autonomously move to the desired destination by exploiting the bearing angle of feature points matched in panoramic images [6]. Polar coordinates of landmarks in omnidirectional images can be used as inputs for localization in a pose-based control

R. Tatsambon Fomena is with Université de Rennes 1, IRISA, Campus de Beaulieu, 35042, Rennes-cedex, France rtatsambeirisa.fr

A. Cherubini and F. Chaumette are with INRIA Rennes-Bretagne Atlantique, IRISA, Campus de Beaulieu, 35042, Rennes-cedex, France acherubi@irisa.fr, chaumett@irisa.fr

H. U. Yoon and Seth Hutchinson are with the Beckman Institute, University of Illinois at Urbana-Champaign, 405 North Matthews Ave, Urbana, IL 61801 hyoon24@uiuc.edu, seth@uiuc.edu scheme [7]. Spherical coordinates of points can be used to keep a vehicle on a predefined path using a conventional camera mounted on a pan-tilt head and an omnidirectional camera [8]: the control using spherical coordinates has been shown to be robust with respect to (w.r.t.) calibration errors on omnidirectional vision systems.

This paper deals with an image-based visual servoing application which consists in positioning a mobile robot w.r.t. a point using an omnidirectional vision system. The main problem addressed here is the design of a robust control scheme. Since the decoupling property has a positive impact on the robustness of a control method, using the image of a single point, we propose a decoupled set of features which ensures a satisfactory robot behavior even in the case of calibration errors in the robotic platform. The second section describes the robotic platform and the type of omnidirectional vision system that can be used as well. In the latter part of Section II the basic concepts of visual servoing are reviewed succinctly. Section III exploits the work in [8] and presents the choice of the set of features used to control the robot from an initial image to a final image. The set decouples the control of the robot rotation from the robot translation. Although the proposed set does not control the pose of the robot, the set enables a simple pure rotation of the robot around its inertia axis which is not the case of the epipolar geometry-based control method proposed in [3]. In Section IV, using the selected set of features, a classical control law is shown to be robust in the presence of point range errors. Finally, Section V presents real experiments that validate the robustness of the proposed decoupled control scheme w.r.t. both point range and calibration errors.

\section{SYSTEM CHARACTERISTICS}

\section{A. Mobile robot characteristics}

This work focuses on a non-holonomic robot (which is a differential-drive robot) with the kinematics of unicycle type (see Fig. 1(a)). As shown in Fig. 1(b), the two control inputs available are: $\mathbf{v}_{r}=\left(v_{r}, \omega_{r}\right)$ where $v_{r}$ is the linear velocity and $\omega_{r}$ is the angular velocity. The robot moves in a planar workspace. Let $\mathcal{F}_{w}=(\mathcal{W}, \mathbf{x}, \mathbf{y}, \mathbf{z})$ be the world frame. The robot configuration is given by $q=\left(r_{x}, r_{y}, r_{\theta}\right)$, where $\left(r_{x}, r_{y}\right)$ is the Cartesian position of the robot center in $\mathcal{F}_{w}$, and $r_{\theta}$ is the robot orientation w.r.t. the world frame $\mathrm{x}$ axis (see Fig. 1(b)). The state equations of the robot are given by

$$
\begin{aligned}
& \dot{r}_{x}=v_{r} \cos r_{\theta} \\
& \dot{r}_{y}=v_{r} \sin r_{\theta} \\
& \dot{r}_{\theta}=\omega_{r}
\end{aligned}
$$




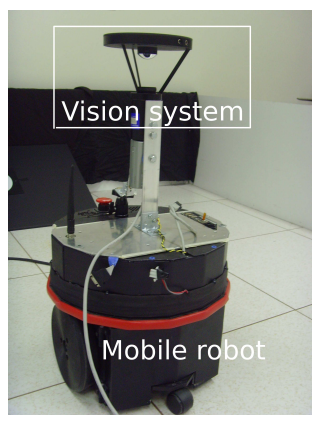

(a)

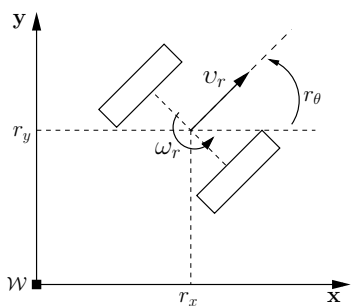

(b)
Fig. 1. Visual servoing system. (a) Super scout mobile robot equipped with a central catadioptric vision system. (b) Robot relevant variables in the plane.

As shown in Fig. 1(a) and Fig. 2(a), the robot senses its environment through a wide FOV vision system called central catadioptric vision system. The next subsection briefly presents the principle and the projection model of this type of vision systems.

\section{B. Central catadioptric vision systems}

A central catadioptric vision system consists of a couple (camera, mirror) which has a single viewpoint [9]. Fig. 2 presents the usual couples (camera, mirror) where the image of an object consists of the camera image (red rays) of the reflection (blue rays) of the object into the mirror.

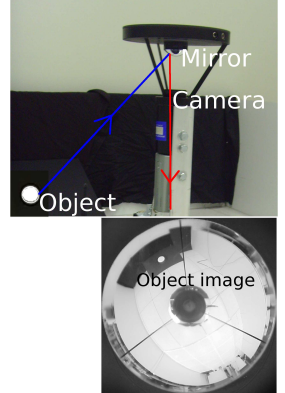

(a)

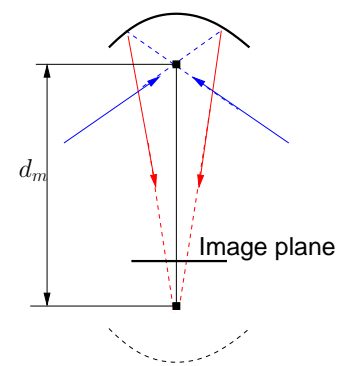

(c)

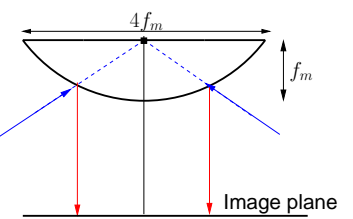

(b)

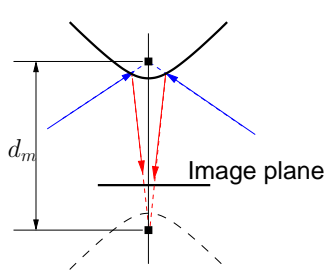

(d)
Fig. 2. Usual couples (camera, mirror). (a) Example of a catadioptric image formation. (b) Paraboloidal mirror and orthographic camera. (c) Ellipsoidal mirror and perspective camera. (d) Hyperboloidal mirror and perspective camera. $f_{m}$ is the mirror focal length and $d_{m}$ is the distance between the focal points.

The central catadioptric image of an object can be decomposed in two steps according to the unified projection model of catadioptric image formation [10]. Fig. 3(a) shows the general case of the central catadioptric projection of a feature

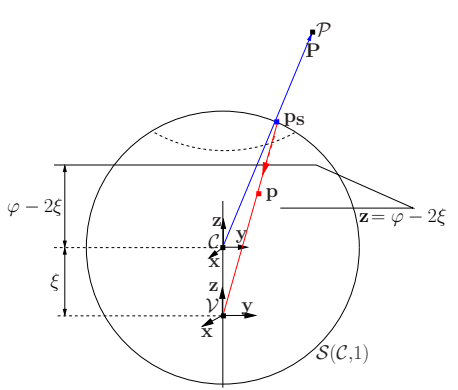

(a)

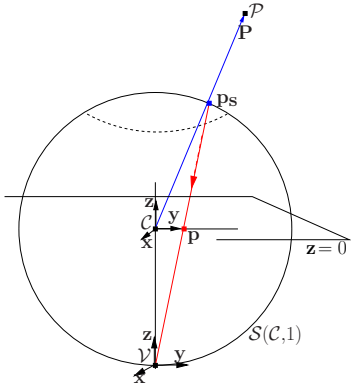

(b)
Fig. 3. Central catadioptric image of a point. (a) General case, cut made perpendicular to the image plane. (b) Paracatadioptric model with $\xi=1$.

point $\mathcal{P}$, where parameters $\varphi>0$ and $\xi \in[0,1]$ describe the mirror shape (see Table I for usual mirrors [11]). The paracatadioptric case, shown in Fig. 3(b) corresponds to the coupling of an orthographic camera and a paraboloidal mirror described by $\xi=1$. Let $\mathcal{F}_{v}$ be the sensor frame $(\mathcal{V}, \mathbf{x}, \mathbf{y}, \mathbf{z})$ and $\mathcal{F}_{c}$ be the center of projection frame $(\mathcal{C}, \mathbf{x}, \mathbf{y}, \mathbf{z})$ where $\mathcal{C}$ is the unique viewpoint of the vision system. The first step is the spherical projection of $\mathcal{P}$ onto the unit sphere $\mathcal{S}(\mathcal{C}, 1): \mathbf{p}_{\mathbf{s}}=\mathbf{P} /\|\mathbf{P}\|$ where $\mathbf{P}$ is the vector coordinates of $\mathcal{P}$. The point $\mathbf{p}_{\mathbf{s}}$ is then expressed in the sensor frame $\mathcal{F}_{v}$ and projected onto the catadioptric image plane $\mathbf{z}=\varphi-2 \xi$ as follows:

$$
p_{x}=p_{s_{x}} /\left(p_{s_{z}}+\xi\right), \quad p_{y}=p_{s_{y}} /\left(p_{s_{z}}+\xi\right)
$$

where $\mathbf{p}=\left(p_{x}, p_{y}\right)$ is the vector coordinates of the projection of $\mathbf{p}_{\mathbf{s}}=\left(p_{s_{x}}, p_{s_{y}}, p_{s_{z}}\right)$. Note here that we consider a unit sensor focal length, that is $\varphi-\xi=1$.

TABLE I

PARAMETERS OF USUAL MIRRORS.

\begin{tabular}{|c|c|c|}
\hline Mirror type & $\xi$ & $\varphi$ \\
\hline \hline Paraboloidal & 1 & $1+2 f_{m}$ \\
\hline Hyperboloidal & $\frac{d_{m}}{\sqrt{d_{m}^{2}+4 f_{m}^{2}}}$ & $\frac{d_{m}+2 f_{m}}{\sqrt{d_{m}^{2}+4 f_{m}^{2}}}$ \\
\hline Ellipsoidal & $\frac{d_{m}}{\sqrt{d_{m}^{2}+4 f_{m}^{2}}}$ & $\frac{d_{m}-2 f_{m}}{\sqrt{d_{m}^{2}+4 f_{m}^{2}}}$ \\
\hline
\end{tabular}

In the next section we present a visual servoing scheme corresponding to our robotic system.

\section{Visual servo control}

We recall that the interaction matrix $\mathbf{L}_{\mathbf{s}} \in \mathbb{R}^{n \times 6}$ related to a set of features $\mathbf{s} \in \mathbb{R}^{n}$ is defined such that $\dot{\mathbf{s}}=\mathbf{L}_{\mathbf{s}} \mathbf{v}_{c}$ where $\mathbf{v}_{c}=(\boldsymbol{v}, \boldsymbol{\omega}) \in \mathfrak{s e}(\mathbf{3})$ is the instantaneous camera velocity [12]: $\boldsymbol{v}=\left(v_{x}, v_{y}, v_{z}\right)$ and $\boldsymbol{\omega}=\left(\omega_{x}, \omega_{y}, \omega_{z}\right)$ are respectively the translational and the rotational velocities of the camera and $\mathfrak{s e}(\mathbf{3}) \simeq \mathbb{R}^{3} \times \mathbb{R}^{3}$ is the Lie algebra of the Lie group of displacements $\mathbf{S E}(\mathbf{3})$.

Visual servoing consists in using data provided by a vision sensor to control the motion of a dynamic system [13]. The principle of visual servoing is to regulate $\mathbf{e}=\mathbf{s}-\mathbf{s}^{*}$, which is the error between a current set of visual features $\mathbf{s}$ and $\mathbf{a}$ desired set of features $\mathbf{s}^{*}$, to zero. In the center of projection 
frame $\mathcal{F}_{c}$, the regulation can be done using the classical control law

$$
\mathbf{v}_{c}=-\lambda \widehat{\mathbf{L}}_{\mathbf{s}}^{+}\left(\mathbf{s}-\mathbf{s}^{*}\right)
$$

where $\lambda$ is a positive gain and $\widehat{\mathbf{L}}_{\mathbf{s}}^{+}$is the pseudo-inverse of an approximation of the interaction matrix related to $\mathbf{s}$.

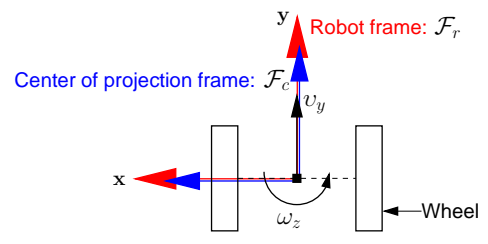

Fig. 4. Robot and center of projection frames in the ideal case.

In this paper, we consider a positioning task with respect to a point. For our robotic platform we assume that a vision system can be ideally placed on top of the robot such that the center of projection frame $\mathcal{F}_{c}$ and the robot frame $\mathcal{F}_{r}$ are aligned as shown on Fig. 4. In this case, since the vision system is rigidly attached to the robot, the linear and the angular velocities of the robot correspond to the translation along the $\mathrm{y}$-axis $v_{y}$ and the rotation about the z-axis $\omega_{z}$ respectively (see Fig. 4):

$$
v_{r}=v_{y}, \omega_{r}=\omega_{z} .
$$

Therefore the appropriate interaction matrix for our visual servoing system is given by

$$
\mathbf{L}_{\mathbf{s}}=\left[\begin{array}{ll}
\mathbf{L}_{v_{y}} & \mathbf{L}_{\omega_{z}}
\end{array}\right]
$$

Since the image of a point enables only to control two DOFs, it is not possible to control the pose of the robot and the camera velocity sent to the low level robot controller is given by

$$
\mathbf{v}_{c}=\mathbf{v}_{r}=-\lambda \widehat{\mathbf{L}}_{\mathbf{s}}^{-1}\left(\mathbf{s}-\mathbf{s}^{*}\right)
$$

In the next section, we choose a set of two features that decouples the control of the robot two degrees of freedom (DOFs).

\section{FEATURE MODELING}

It is possible to use the two coordinates $\mathbf{p}=\left(p_{x}, p_{y}\right)$ of the catadioptric image of a point to control the robot two DOFs $v_{y}$ and $\omega_{z}$. However the corresponding interaction matrix, obtained from the six DOFs case in [14], is highly coupled:

$$
\mathbf{L}_{\mathbf{p}}=\left[\begin{array}{cc}
\frac{\xi p_{x} p_{y}}{\|\mathbf{P}\|} & p_{y} \\
-\frac{1+p_{x}^{2}+p_{y}^{2}\left(1-\xi\left(\xi+\lambda_{\xi}\right)\right)}{\|\mathbf{P}\|\left(\xi+\lambda_{\xi}\right)} & -p_{x}
\end{array}\right]
$$

where $\xi$ is the mirror parameter and $\lambda_{\xi}=\sqrt{1+\left(1-\xi^{2}\right)\left(p_{x}^{2}+p_{y}^{2}\right)}$.

In the case of a pure rotation of the robot around its inertia axis, the interaction matrix (7) induces also a translation of the robot which is not desirable. Indeed, for a $180^{\circ}$ rotation around the optical axis of a six DOFs manipulator, using the coordinates of the perspective image of several points leads to an unachievable motion of the robot [15]. This is the reason why a decoupled control is desirable. The decoupled control can be obtained using a partition approach [16], cylindrical coordinates [17], moment invariants [18] or spherical moments [19].

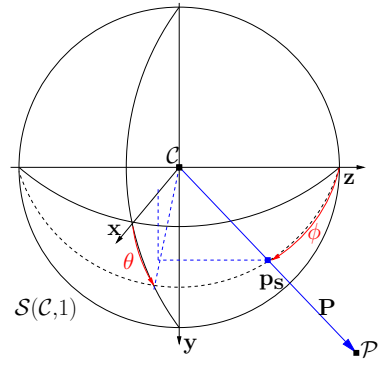

Fig. 5. Spherical projection of a point.

A decoupled interaction matrix can be obtained using the spherical projection of the point object. As shown in Fig. 5, a point $\mathbf{p}_{\mathbf{s}}=\left(p_{s x}, p_{s y}, p_{s z}\right)$ on a sphere can be minimally parameterized using its spherical coordinates

$\mathbf{s}=(\phi, \theta)$, with $\phi=\arccos \left(p_{s z}\right)$ and $\theta=\arctan \left(p_{s y} / p_{s x}\right)$.

By exploiting the coordinates $\mathbf{p}=\left(p_{x}, p_{y}\right)$ of the catadioptric image of the point, it is possible to compute features $\mathbf{s}$. Indeed by expressing $\mathbf{p}_{\mathbf{s}}$ as a function of $\mathbf{p}$ from (2) and by using (8), it is possible to show that

$$
\phi=\arccos \left(\frac{\xi+\lambda_{\xi}}{p_{x}^{2}+p_{y}^{2}+1}-\xi\right), \theta=\arctan \left(\frac{p_{y}}{p_{x}}\right)
$$

where $\xi$ and $\lambda_{\xi}$ are defined in (7). From (9), it is important to note that the set $\mathbf{s}$ does not have any singularity in the image space. Indeed the configuration where $p_{x}=p_{y}=0$ in the catadioptric image plane corresponds to the center of the dead region in the image space (see Fig. 2(a)), and the dead region is (of course) not included in the region where the object is visible.

The pose-based control of a mobile robot using spherical coordinates of several points has been shown to be robust to calibration errors on an omnidirectional vision system in a vehicle prerecorded-path following application [8]. In [8], the interaction matrix relating the change of $\mathbf{s}$ to the change of motion in plane $\left(v_{x}, v_{y}\right.$ and $\left.\omega_{z}\right)$ was presented. In this paper we do not control the pose of the robot; the appropriate interaction matrix, related to motions $v_{y}$ and $\omega_{z}$, is given by

$$
\mathbf{L}_{\mathbf{s}}=\left[\begin{array}{cc}
l_{v} & 0 \\
l_{\omega, v} & -1
\end{array}\right]
$$

where

$$
\left\{\begin{array}{l}
l_{v}=-(\sin \theta \cos \phi) /\|\mathbf{P}\| \\
l_{\omega, v}=-\cos \theta /(\|\mathbf{P}\| \sin \phi),
\end{array}\right.
$$

with $\|\mathbf{P}\|$ the range or distance of the point from the center of projection $\mathcal{C}$.

Note that $\sin \phi \neq 0$ in the object visibility region since $\sin \phi=0$ is equivalent to $p_{s_{x}}=p_{s_{y}}=0$ (see Fig. 5), which corresponds to center $p_{x}=p_{y}=0$ of the dead region in the catadioptric image plane. 
Taking the inverse of the interaction matrix (10) and plugging it into (6) leads to the ideal control law

$$
\left\{\begin{array}{l}
v_{y}=-\lambda \frac{1}{l_{v}}\left(\phi-\phi^{*}\right) \\
\omega_{z}=l_{\omega, v} v_{y}+\lambda\left(\theta-\theta^{*}\right) .
\end{array}\right.
$$

The decoupling in $\mathbf{L}_{\mathbf{s}}$ shows that feature $\theta$ is the only feature which is sensitive to the robot rotation around its inertia axis. Indeed, for a pure rotation of the robot, the value of $\phi$ is constant, i.e. $\phi=\phi^{*}$, from (11) we obtain

$$
v_{y}=0, \omega_{z}=\lambda\left(\theta-\theta^{*}\right) .
$$

In addition, in this case (pure rotation), there is no singularity of the control (12) in the visibility space since the value $\theta$ is always defined when the object point is visible.

It is important to note that contrary to the work done in [3] where the image of several points is used, we control neither the pose of the robot nor the non-holonomic constraint since we use the image of a single point. However a simple pure rotation between the current and the desired images is impossible to realize using the epipolar geometry-based control law proposed in [3]. This is due to the fact that the epipoles are not defined in this case.

The interaction matrix (10) loses its rank 2 if and only if $l_{v}=0$ that is $\phi=(2 k+1) \pi / 2$ or $\theta=k \pi$ with $k \in \mathbb{Z}$. This corresponds to the cases where the point $\mathcal{P}$ is such that either $P_{z}=0$ or $P_{y}=0$. Using (2), it is possible to show that the region of rank loss in the image space is defined as follows:

$$
\mathcal{R}_{1}=\left\{\left(p_{x}, p_{y}\right), p_{y}=0 \text { or } p_{x}^{2}+p_{y}^{2}=1 / \xi^{2}\right\} .
$$

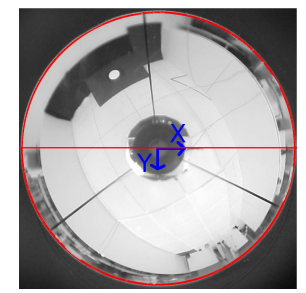

Fig. 6. Region $\mathcal{R}_{1}$ in red.

An illustration of $\mathcal{R}_{1}$ is given on Fig. 6 where we can see that the circle is far from the exploitable space in the image plane. Altough the line $p_{y}=0$ is a serious issue, it is possible to deal with it easily. Indeed by first controlling $\omega_{z}$ using the control law (12), it is always possible to position the robot so that the image of the point lies in the adequate half image plane (see Fig. 6) before controlling both $v_{y}$ and $\omega_{z}$ simultaneously in the image space where $\mathbf{L}_{\mathbf{s}}$ is always of rank 2. Note also that the loss of rank on the line $p_{y}=0$ is due to the nature of our non-holonomic robot, which can be controlled with only two inputs ( $v_{x}$ motion is impossible).

In the next section we analyse the stability of the proposed control scheme.

\section{ROBUSTNESS ANALYSIS}

The interaction matrix (10) depends on the point range $\|\mathbf{P}\|$ from the center of projection. Since the value of $\|\mathbf{P}\|$ is unknown in practice, we use an estimated value $\widehat{\|\mathbf{P}\|}$ which can be expressed as follows

$$
\widehat{\|\mathbf{P}\|}=\widehat{\left|P_{z}\right|} \sqrt{\left(P_{x} / P_{z}\right)^{2}+\left(P_{y} / P_{z}\right)^{2}+1}
$$

where $P_{x} / P_{z}=p_{s x} / p_{s z}$ and $P_{y} / P_{z}=p_{s y} / p_{s z}$ can be measured, using (2), from the catadioptric image plane.

This section aims at analyzing the robustness of the control (11) w.r.t. point range estimation errors, that is errors on the estimation $\|\widehat{\mathbf{P}}\|$. In the following, we derive a sufficient condition for the robustness of the control in the image space.

Here we assume that the interaction matrix never loses its rank during the servoing, that is we consider the image space where $\mathbf{L}_{\mathbf{s}}$ is always of rank 2 . We also suppose that the point object is visible all times. Assuming that we do not have neither image processing errors nor vision system calibration errors, the closed-loop system equation (using the control law (11)) can be written as:

$$
\dot{\mathbf{e}}=-\lambda \mathbf{L}_{\mathbf{s}} \widehat{\mathbf{L}}_{\mathbf{s}}^{-1} \mathbf{e}
$$

with $\mathbf{e}=\mathbf{s}-\mathbf{s}^{*}$,

$$
\widehat{\mathbf{L}}_{\mathbf{s}}^{-1}=\left[\begin{array}{cc}
1 / \widehat{l}_{v} & 0 \\
\widehat{l}_{\omega, v} / \widehat{l}_{v} & -1
\end{array}\right],
$$

where $\widehat{l}_{v}=-(\sin \theta \cos \phi) / \widehat{\|\mathbf{P}\|}, \widehat{l}_{\omega, v}=-\cos \theta /(\widehat{\|\mathbf{P}\|} \sin \phi)$.

The stability of the system (15) can be analysed: in the ideal case (no errors), (15) becomes $\dot{\mathbf{e}}=-\lambda \mathbf{e}$ which means that the system is globally asymptotically stable (GAS). Indeed no trajectory will cause feature point to pass into the dead zone since in the ideal case the system will always execute the shortest path, i.e. a geodesic on a sphere, from the initial to the desired $\left(\mathbf{s}^{*}=\left(\phi^{*}, \theta^{*}\right)\right)$ configurations.

In the case of error on $\widehat{\|\mathbf{P}\|}$, the robustness domain of the control is given by the theorem 1 below. Of course there is no guarantee that the feature point trajectory will not pass into the dead zone, but this visibility issue can easily be dealt with by using path-planning techniques in the image [20].

Theorem 1: the equilibrium point $\mathbf{e}=0$ of the system (15) is GAS in the image space for all range estimates $\|\widehat{P}\|$ that satisfy

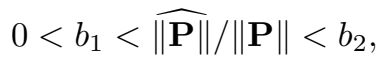

with $\quad b_{1}=1+\frac{2-2 \sqrt{a^{2}+1}}{a^{2}}, \quad b_{2}=1+\frac{2+2 \sqrt{a^{2}+1}}{a^{2}} \quad$ where $a=-\cos \theta /(\sin \phi \sin \theta \cos \phi)$.

The proof of the theorem 1, given in [21], uses the following lemma:

Lemma 1: the equilibrium point $\mathbf{e}=0$ of the system (15) is GAS in the image space if the symmetric part $\mathbf{M}_{\mathbf{s}}=\left(\mathbf{L}_{\mathbf{s}} \widehat{\mathbf{L}}_{\mathbf{s}}^{-1}+\left(\mathbf{L}_{\mathbf{s}} \widehat{\mathbf{L}}_{\mathbf{s}}^{-1}\right)^{\top}\right) / 2$ of $\mathbf{L}_{\mathbf{s}} \widehat{\mathbf{L}}_{\mathbf{s}}^{-1}$ is positive definite.

Proof: [Lemma 1] From Lyapunov theory, the equilibrium point $\mathbf{e}=0$ of the system (15) is asymptotically stable if $\mathbf{L}_{\mathbf{s}} \widehat{\mathbf{L}}_{\mathbf{s}}^{-1}>0$. The real matrix $\mathbf{L}_{\mathbf{s}} \widehat{\mathbf{L}}_{\mathbf{s}}^{-1}$ is given by

$$
\mathbf{L}_{\mathbf{s}} \widehat{\mathbf{L}}_{\mathbf{s}}^{-1}=\left[\begin{array}{cc}
\widehat{\|\mathbf{P}\| /\|\mathbf{P}\|} & 0 \\
a(1-\widehat{\|\mathbf{P}\| /\|\mathbf{P}\|)} & 1
\end{array}\right]
$$


where $a=-\cos \theta /(\sin \phi \sin \theta \cos \phi)$.

Since we consider the image space region where the current interaction matrix $\mathbf{L}_{\mathbf{s}}$ is always of rank 2 , that is either $\mathbf{y}<0$ or $\mathbf{y}>0$ (see Fig. 6), the determinant of $\mathbf{L}_{\mathbf{s}} \widehat{\mathbf{L}}_{\mathbf{s}}^{-1}$ given by $\|\widehat{\mathbf{P}}\| /\|\mathbf{P}\|$ is never nul $\left(\left|\mathbf{L}_{\mathbf{s}} \widehat{\mathbf{L}}_{\mathbf{s}}^{-1}\right| \neq 0\right)$. In this case, the equilibrium point $\mathbf{e}=0$ is unique. In addition if $\mathbf{L}_{\mathbf{s}} \widehat{\mathbf{L}}_{\mathbf{S}}^{-1}>0$ then for a fixed desired position of the point in the image space $\mathbf{y}<0$ (respectively $\mathbf{y}>0$ ) the control converges for any initial position of the point in the image space $\mathbf{y}<0$ (respectively $\mathbf{y}>0$ ). Therefore if $\mathbf{L}_{\mathbf{s}} \widehat{\mathbf{L}}_{\mathbf{s}}^{-1}>0$, the asymptotic stability of the equilibrium $\mathbf{e}=0$ is global in the considered image space.

Since the real matrix $\mathbf{L}_{\mathbf{s}} \widehat{\mathbf{L}}_{\mathbf{s}}^{-1}$ is not symmetric, $\mathbf{L}_{\mathbf{s}} \widehat{\mathbf{L}}_{\mathbf{s}}^{-1}$ is positive definite if and only if its symmetric part $\mathbf{M}_{\mathbf{s}}=\left(\mathbf{L}_{\mathbf{s}} \widehat{\mathbf{L}}_{\mathbf{s}}^{-1}+\left(\mathbf{L}_{\mathbf{s}} \widehat{\mathbf{L}}_{\mathbf{s}}^{-1}\right)^{\top}\right) / 2$ is positive definite.

Therefore, a sufficient condition for the global asymptotic stability of the equilibrium point $\mathbf{e}=0$ is given by the strict positiveness of the symmetric matrix $\mathbf{M}_{\mathbf{s}}$ of $\mathbf{L}_{\mathbf{s}} \widehat{\mathbf{L}_{\mathbf{S}}}-1$.

As long as the ratio $\widehat{\|\mathbf{P}\|} /\|\mathbf{P}\|$ lies in the robustness domain given by the above theorem, for a fixed desired position of the point in the image space $\mathbf{y}>0$ (respectively $\mathbf{y}<0$ ) (see Fig. 6), the control converges from any initial position of the point in the image space $\mathbf{y}>0$ (respectively $\mathbf{y}<0$ ). From a practical point of view the value of $\|\widehat{P}\|$ should not be set constant. Indeed the robustness domain to point range errors is constrained by the values of $\phi$ and $\theta$. For example, if $\theta=\phi=\pi / 4$ then $a=-1 / 2$ and $0.056<\widehat{\| \mathbf{P}}\|/\| \mathbf{P} \|<17.94$. In the next section, we will show that the control converges for large errors between the initial and final images in presence of both point range and calibration errors.

\section{EXPERIMENTAL RESULTS}

This section presents relevant experimental results that validate the robustness of our approach to both modeling and calibration errors on the visual servoing system. The vision system intrinsic parameters (principal point coordinates and focal lengths), obtained using the calibration method proposed in [22], are given by $u_{0}=693.60, v_{0}=453.14$, $f_{u}=388.40$ and $f_{v}=388.67$.

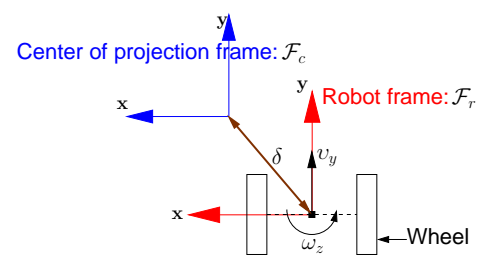

Fig. 7. Robot and center of projection frames in practice.

In order to validate the robustness of our approach to the robot calibration, we have set rough values for the parameters of the low level controller of the robot and we have introduced a misalignment between the robot and the center of projection frames (see Fig. 7): the shift between these frames has been roughly estimated to $\delta=1 \mathrm{~cm}$.

In addition, for the estimation of $\widehat{\|\mathbf{P}\|}$ (see (14)), the value of $\widehat{\left|P_{z}\right|}$ has been set to the constant value $0.8 \mathrm{~m}$ which roughly corresponds to the desired depth $\widehat{\left|P_{z}^{*}\right|}$. Except for one particular experiment, the value of the gain of the control (6) has been set to $\lambda=0.1$.

The desired set of features $\mathbf{s}^{*}$ has been defined by moving the robot. Five different initial positions of the robot have been selected. The behavior of the robot illustrated on the figures below validates the robustness of the decoupled scheme to point range and robot calibration errors.

In the first experiment, it is clear from Fig. 8(c)that the final position of the robot corresponds to the desired position of the point in the image. Despite the large and abrupt variations on the visual features $\theta$ ( see Fig. 8(c)), the control sent to the robot does not present oscillations as shown on Fig. 8(d). The video attached to this paper displays the first experiment.

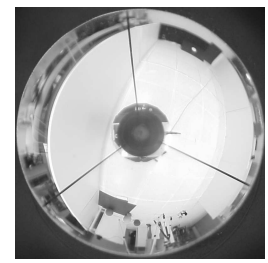

(a)

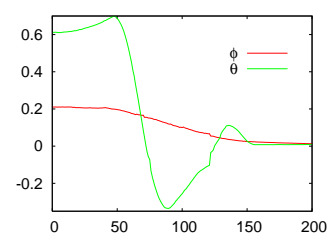

(c)

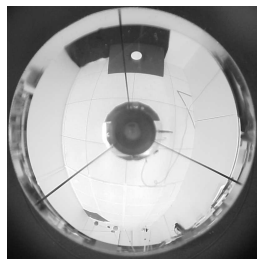

(b)

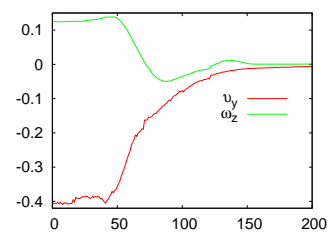

(d)
Fig. 8. First case. (a) Initial image. (b) Final image. (c) Errors on s (rad). (d) Camera velocities $(\mathrm{m} / \mathrm{s}$ and $\mathrm{rad} / \mathrm{s})$.

The abrupt variation on feature $\theta$ is due to the low compensation on the angular velocity $\omega_{z}$ since $l_{v}$ and $l_{\omega, v}$ are roughly estimated (see (11)). The low compensation of the angular velocity $\omega_{z}$ can also be explained by the rough approximation of the parameters of the robot low level controller. For large rotation motions of the robot, the abrupt variations of feature $\theta$ could cause the failure of visual servoing. We have therefore set the gain of the control to $\lambda=0.05$ for the second case as shown in Fig. 9. In the second case, note that the initial image of the point is almost in the region where $\mathbf{L}_{\mathbf{s}}$ can lose its rank (see the line in Fig. 9(a)). The experiment shows a more smooth variation on the errors (see Fig. 9(c)) and the velocities (see Fig. 9(d)). More importantly note also in this case that we have used a rough approximation of $\| \widehat{\mathbf{P} \|}$ while when $\theta$ tends to 0 (i.e. both $b_{1}$ and $b_{2}$ tend to 1 ), from Theorem 1 the control is GAS if the value of $\|\widehat{P}\|$ is accurately estimated. The robustness domain to errors on the range estimate is thus larger than the one given by Theorem 1 . 
For the last experiment, we have added the following errors on the vision system intrinsic parameters: $\widehat{u}_{0}=u_{0}-15 \% u_{0}, \widehat{v}_{0}=v_{0}+17 \% v_{0}, \widehat{f}_{u}=f_{u}+10 \% f_{u}$ and $\widehat{f}_{v}=f_{v}+20 \% f_{v}$. Once again the control converges as shown on Fig. 10(c) and 10(d).

To sum up, the decoupled control scheme proposed is robust with respect to both calibration and point range errors.

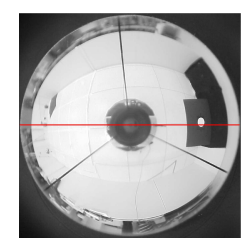

(a)

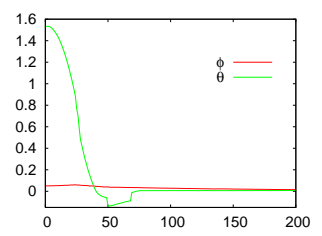

(c)

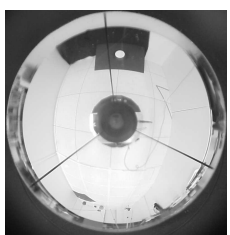

(b)

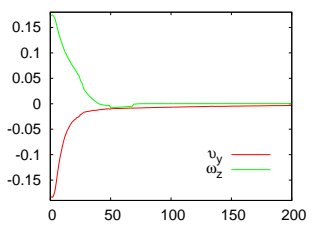

(d)
Fig. 9. Second case. (a) Initial image. (b) Final image. (c) Errors on $\mathbf{s}$ (rad). (d) Camera velocities $(\mathrm{m} / \mathrm{s}$ and $\mathrm{rad} / \mathrm{s})$.

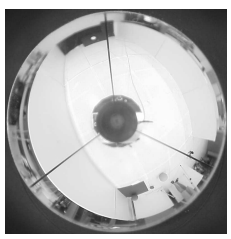

(a)

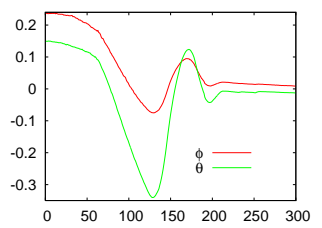

(c)

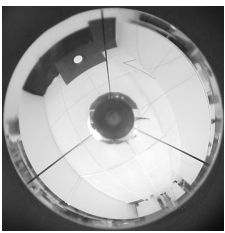

(b)

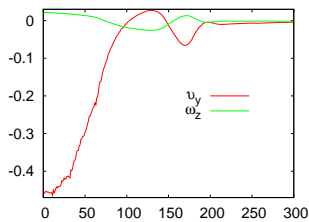

(d)
Fig. 10. Third case: (a) Initial image. (b) Final image. (c) Errors on $\mathbf{s}$ (rad). (d) Camera velocities (m/s and $\mathrm{rad} / \mathrm{s})$.

\section{CONCLUSIONS AND FUTURE WORKS}

In this paper we have proposed a decoupled scheme for a robust vision-based control of a mobile robot equipped with a catadioptric vision system. More precisely, the spherical coordinates of a point have been used to design a decoupled visual servoing scheme to position a unicycle with respect to an object point. Using the new set of features, the control has been theoretically proved to be GAS in the ideal case, and robust in the presence of error in the range of the object point. In practice we have validated the robustness of the control w.r.t. both point range and calibration errors. Experimental results demonstrate successful performance. In future work, it would be interesting to use image path-planning techniques to keep the feature point in the non-dead zone in the case of error on the estimated range of the object point.

\section{ACKNOWLEDGMENTS}

The authors would like to thank J. Davidson and S. Candido for their help during experiments.

This work was funded by the INRIA-TALISKER project.

\section{REFERENCES}

[1] L. Delahoche, C. Pegard, B. Marhic, and P. Vasseur, "A navigation system based on an omnidirectional vision sensor," in IEEE/RSJ Int. Conf. on Intelligent Robots and Systems, IROS'97, pp. 718-724.

[2] G. Blanc, Y. Mezouar, and P. Martinet, "Indoor navigation of a wheeled mobile robot along visual routes," in IEEE Int. Conf. on Robotics and Automation, 2005.

[3] G. Mariottini, G. Oriolo, and D. Prattichizzo, "Image-based visual servoing for nonholonomic mobile robots using epipolar geometry," IEEE Trans. on Robotics, vol. 23, no. 1, pp. 87-100, February 2007.

[4] A. Diosi, A. Remazeilles, S. Segvic, and F. Chaumette, "Outdoor visual path following experiments," in IEEE/RSJ Int. Conf. on Intelligent Robots and Systems, 2007.

[5] S. S. Jones, C. Andersen, and J. L. Crowley, "Appearance based processes for visual navigation," in IEEE/RSJ Int. Conf. on Intelligent Robots and Systems, 1997.

[6] A. A. Argyros, K. E. Bekris, S. C. Orphanoudakis, and L. E. Kavraki, "Robot homing by exploiting panoramic vision," Autonomous Robots, vol. 19, no. 1, pp. 7-25, 2005.

[7] K. Usher, P. Ridley, and P. Corke, "Visual servoing of a car-like vehicle - an application of omnidirectional vision," in IEEE Int. Conf. on Robotics and Automation, 2003.

[8] D. Burschka and G. Hager, "Vision-based control of mobile robots," in IEEE Int. Conf. on Robotics and Automation, 2001.

[9] S. Baker and S. Nayar, "A theory of catadioptric image formation," in IEEE Int. Conf. on Computer Vision, Jan. 1998, pp. 35-42.

[10] C. Geyer and K. Daniilidis, "A unifying theory for central panoramic systems and practical implications," European Conference on Computer Vision, vol. 29, pp. 159-179, May 2000.

[11] J. Barreto and H. Araujo, "Geometric properties of central catadioptric line images," in ECCV, Copenhagen, Denmark, May 2002.

[12] B. Espiau, F. Chaumette, and P. Rives, "A new approach to visual servoing in robotics," IEEE Trans. on Robotics and Automation, vol. 8, no. 3, pp. 313-326, Jun. 1992.

[13] S. Hutchinson, G. Hager, and P. Corke, "A tutorial on visual servo control," IEEE Trans. on Robotics and Automation, vol. 12, no. 3, pp. 651-670, Oct. 1996.

[14] J. Barreto, F. Martin, and R. Horaud, "Visual servoing/tracking using central catadioptric images," in Int. Symposium on Experimental Robotics, Ischia, Italy, Jul. 2002.

[15] F. Chaumette, "Potential problems of stability and convergence in image-based and position-based visual servoing," in The Confluence of Vision and Control, D. Kriegman, G. Hager, and A. S. Morse, Eds. LNCIS Series, No 237, Springer-Verlag, 1998, pp. 66-78.

[16] P. Corke and S. Hutchinson, "A new partitioned approach to imagebased visual visual servo control," IEEE Trans. on Robotics and Automation, vol. 17, no. 4, pp. 507-515, Aug. 2001.

[17] M. Iwatsuki and N. Okiyama, "A new formulation for visual servoing based on cylindrical coordinate system," IEEE Trans. on Robotics, vol. 21, no. 2, pp. 266-273, Apr. 2005.

[18] O. Tahri and F. Chaumette, "Point-based and region-based image moments for visual servoing of planar objects," IEEE Trans. on Robotics, vol. 21, no. 6, pp. 1116-1127, Dec. 2005.

[19] T. Hamel and R. Mahony, "Visual servoing of an under-actuated dynamic rigid-body system: an image-based approach," IEEE Trans. on Robotics and Automation, vol. 18, no. 2, pp. 187-198, Apr. 2002.

[20] Y. Mezouar and F. Chaumette, "Path planning for robust image-based control," IEEE Trans. on Robotics and Automation, vol. 18, no. 4, pp. 534-549, Aug. 2002.

[21] R. Tatsambon Fomena, "Asservissement visuel par projection sphérique," Ph.D. dissertation, Université de Rennes 1, Nov. 2008.

[22] C. Mei and P. Rives, "Single view point omnidirectional camera calibration from planar grids," in IEEE Int. Conf. on Robotics and Automation, Rome, Italy, Apr. 2007. 